\section{International Scientific Journal Theoretical \& Applied Science}

p-ISSN: 2308-4944 (print) e-ISSN: 2409-0085 (online)

Year: $2015 \quad$ Issue: 03 Volume: 23

Published: $30.03 .2015 \quad$ http://T-Science.org
Alexandr Nikolayevich Shevtsov candidate of technical sciences, member of PILA (USA),

Department of «Mathematics»,

Deputy Director on Science of faculty of information technologies, automation and telecommunications, Taraz state University named after M.Kh. Dulati, Kazakhstan Shev_AlexXXXX@mail.ru

SECTION 2. Applied mathematics. Mathematical modeling.

\title{
ABOUT DYNAMIC NESTING OF LORENZ ATTRACTORS
}

Abstract: In the study of Lorenz attractors in the system of differential equations is used the first derivative of a function. This study shows the independence of the existence of the attractor from the order of the derivative.

Key words: attractor, Lorenz, derivative, order.

Language: Russian

Citation: Shevtsov AN (2015) ABOUT DYNAMIC NESTING OF LORENZ ATTRACTORS. ISJ Theoretical \& Applied Science 03 (23): 176-179.

Soi: http://s-o-i.org/1.1/TAS*03(23)30 Doi: croskef http://dx.doi.org/10.15863/TAS.2015.03.23.30

\section{О ДИНАМИЧЕСКОЙ ВЛОЖЕННОСТИ АТТРАКТОРОВ ЛОРЕНЦА}

Аннотация: При изучении аттракторов Лоренца в системе дифференциальных уравнений используется первая производная от функиии. В данном исследовании показана независимость существования аттрактора от порядка данной производной.

Ключевые слова: аттрактор, Лорени, производная, порядок.

\section{Введение}

Система дифференциальных уравнений, решение которой, приводит к созданию аттрактора Лоренца [1-9] имеет вид:

$$
\left\{\begin{array}{l}
\dot{x}=\sigma(y-x) \\
\dot{y}=x(r-z)-y \\
\dot{z}=x y-b^{*} z
\end{array}\right.
$$

Вводя обозначения:

$$
\begin{aligned}
& M\left(x_{0}, y_{0}, z_{0}\right) \text { - начальная точка, } \\
& \begin{array}{l}
a=\sigma, \\
b=r, \quad \text { коэффициенты системы, } \\
c=b *
\end{array}
\end{aligned}
$$

рассмотрим численное решение этой системы на промежутке $[-10,10]$. Разобьем интервал на отдельные промежутки с шагом $h=0.0001$.

Методика

Зададим цикл на данном промежутке с шагом $h$, и начиная с точки $M$, будем рассчитывать последовательно все последующие значения системы (1). Получим выражение (2).
Здесь, на каждом последующем шаге, рассчитывается очередная точка решения системы (1) численным методом.

$$
\begin{aligned}
& x_{i+1}=x_{i}+a\left(-x_{i}+y_{i}\right) h, \\
& y_{i+1}=y_{i}+\left(b x_{i}+y_{i}-z_{i} x_{i}\right) h, \\
& z_{i+1}=z_{i}+\left(-c z_{i}+x_{i} y_{i}\right) h .
\end{aligned}
$$

Разработаем алгоритм для построения аттрактора на языке Delphi [8-10].

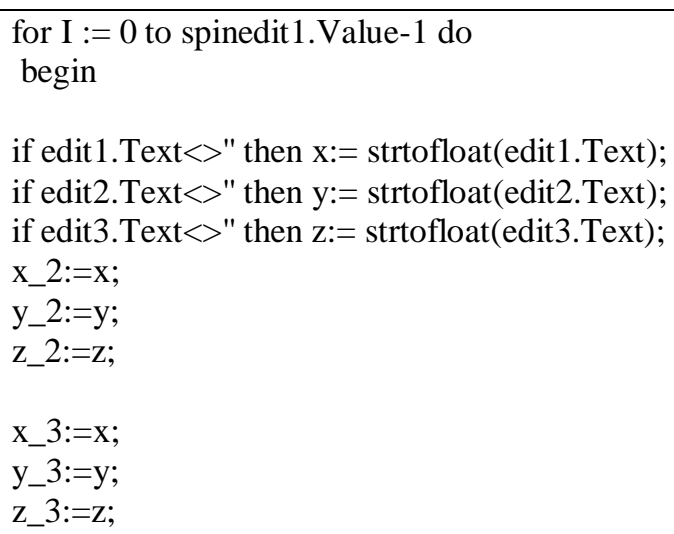


$\mathrm{nn}:=-10$;

while $n n<10$ do

begin

$\mathrm{nn}:=\mathrm{nn}+\mathrm{dt}$;

ai: $=0$;

bi: $=0$; ci: $=0$;

case radiogroup1.ItemIndex of

$1:$ ai: $=\mathrm{i} / 1$

$2: \mathrm{bi}:=\mathrm{i} / 1$

$3: \mathrm{ci}:=\mathrm{i} / 1$

end;

$$
\begin{aligned}
& \mathrm{x} 1:=\mathrm{x}+(\mathrm{a}+\mathrm{ai}) *(-\mathrm{x}+\mathrm{y})^{*} \mathrm{dt} \\
& \mathrm{y} 1:=\mathrm{y}+\left((\mathrm{b}+\mathrm{bi}) * \mathrm{x}-\mathrm{y}-\mathrm{z}^{*} \mathrm{x}\right) * \mathrm{dt} \\
& \mathrm{z} 1:=\mathrm{z}+\left(-(\mathrm{c}+\mathrm{ci}) * \mathrm{z}+\mathrm{x}^{*} \mathrm{y}\right) * \mathrm{dt} \\
& \mathrm{x}:=\mathrm{x} 1 ; \\
& \mathrm{y}:=\mathrm{y} 1 ; \\
& \mathrm{z}:=\mathrm{z} 1 ;
\end{aligned}
$$

glcolor $3 \mathrm{f}(\mathrm{Col} 2+\mathrm{i} / 100, \mathrm{i} / 10+\mathrm{Col} 2$

shr $8+0.1, \mathrm{i} / 100+\operatorname{Col} 2 \mathrm{shr} 16+0.1)$;

glVertex3d(x/10,y/10,z/10-1.7);

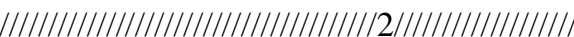

$\mathrm{x} 1:=\mathrm{x} \_2+(\mathrm{a}+\mathrm{ai}) *\left(-\mathrm{x} \_2+\mathrm{y} \_2\right) * \mathrm{dt} *(\mathrm{dt}+2)$

$\mathrm{y} 1:=\mathrm{y} \_2+\left((\mathrm{b}+\mathrm{bi}) * \mathrm{x} \_2-\mathrm{y} \_2-\right.$

$\left.\mathrm{z} \_2 * \mathrm{x} \_2\right) * \mathrm{dt} *(\mathrm{dt}+2)$;

$\mathrm{z} 1:=\mathrm{z} \_2+\left(-(\mathrm{c}+\mathrm{ci}) * \mathrm{z} \_2+\mathrm{x} \_2 * \mathrm{y} \_2\right) * \mathrm{dt} *(\mathrm{dt}+2)$

$\mathrm{x} 2:=\mathrm{x} 1$;

y_2:=y1;

$\mathrm{z} \_2:=\mathrm{z} 1$;

glColor $3 \mathrm{~d}(5,5,0)$;

glVertex3d(x_2/10,y_2/10,z_2/10-1.7);

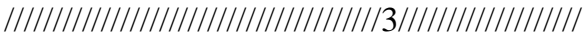

$$
\mathrm{x} 1:=\mathrm{x} \_3+(\mathrm{a}+\mathrm{ai}) *(-
$$

$\left.\mathrm{x} \_3+\mathrm{y} \_3\right) * \mathrm{dt} *(\mathrm{dt} * \mathrm{dt}+3 * \mathrm{dt}+3)$;

$\mathrm{y} 1:=\mathrm{y} \_3+\left((\mathrm{b}+\mathrm{bi}) * \mathrm{x} \_3-\mathrm{y} \_3-\right.$

$\left.\mathrm{z} \_3 * \mathrm{x} \_3\right) * \mathrm{dt} *(\mathrm{dt} * \mathrm{dt}+3 * \mathrm{dt}+3)$;

$\mathrm{z} 1:=\mathrm{z} \_3+(-$

$\left.(\mathrm{c}+\mathrm{ci}) * \mathrm{z} \_3+\mathrm{x} \_3 * \mathrm{y} \_3\right) * \mathrm{dt} *(\mathrm{dt} * \mathrm{dt}+3 * \mathrm{dt}+3)$;

$\mathrm{x} \_3:=\mathrm{x} 1$;

$\mathrm{y} \_3:=\mathrm{y} 1$;

z_3:= z1;

glColor $3 \mathrm{~d}(0,5,0)$;

glVertex3d(x_3/10,y_3/10,z_3/10-1.7);

end;

end;

Полученный аттрактор для параметров рис.1. отображен на рис. 2 .

Варьируя производную получим разностные схемы для второго, и более высоких порядков, рис.3.

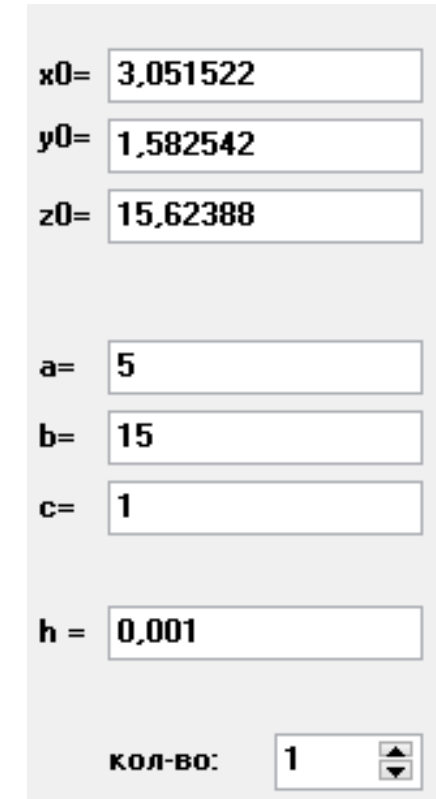

\section{Рисунок 1 - Расчетные параметры.}

Здесь (рис.3) красным отображен график системы - (1), желтым - (3), зеленым, соответственно, - (4). Полученные решения демонстрируют близость самих функций и сохранность аттрактора, независимо от порядка производной.

$$
\begin{aligned}
& \left\{\begin{array}{l}
\frac{\partial^{2} x}{\partial t^{2}}=\sigma(y-x) \\
\frac{\partial^{2} y}{\partial t^{2}}=x(r-z)-y \\
\frac{\partial^{2} z}{\partial t^{2}}=x y-b^{*} z
\end{array}\right. \\
& \left\{\begin{array}{l}
\frac{\partial^{3} x}{\partial t^{3}}=\sigma(y-x) \\
\frac{\partial^{3} y}{\partial t^{3}}=x(r-z)-y \\
\frac{\partial^{3} z}{\partial t^{3}}=x y-b^{*} z
\end{array}\right.
\end{aligned}
$$

Аналогичные расчеты можно продолжить и на более высокие порядки производных, причем свойство аттрактора будет сохраняться. 


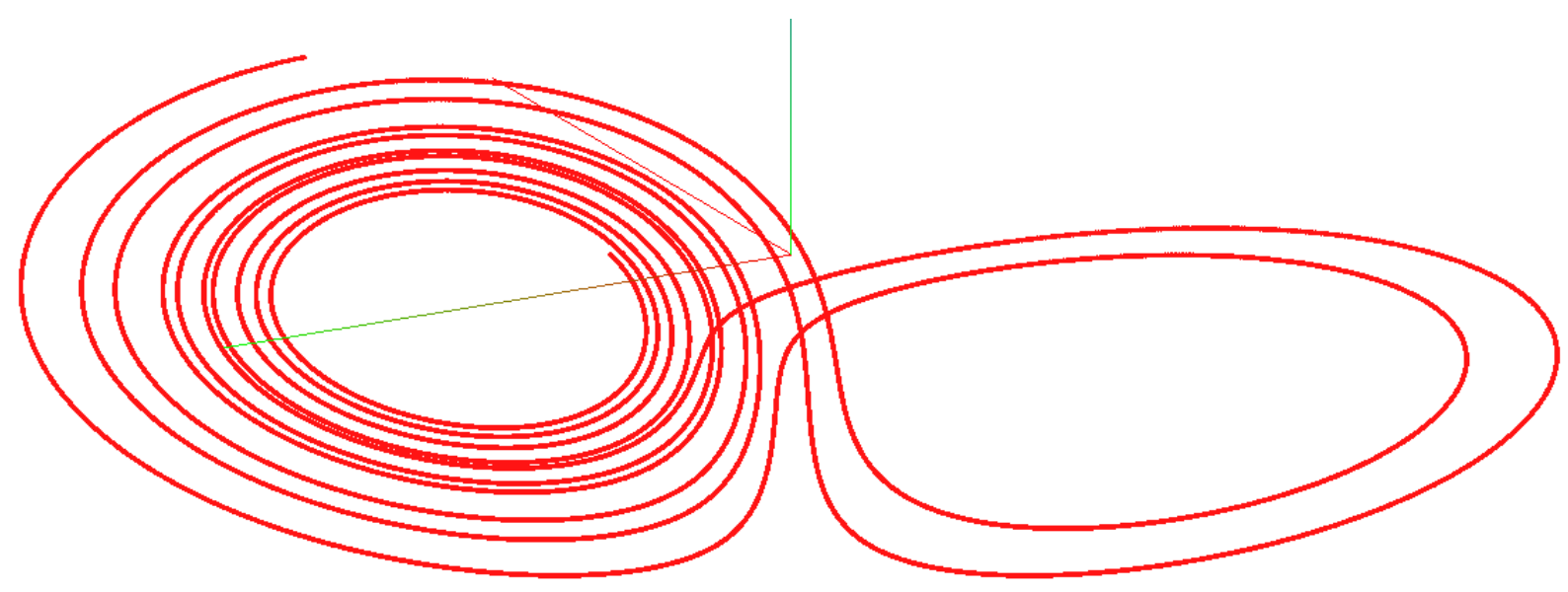

Рисунок 2 - Аттрактор Лоренца (первая производная).
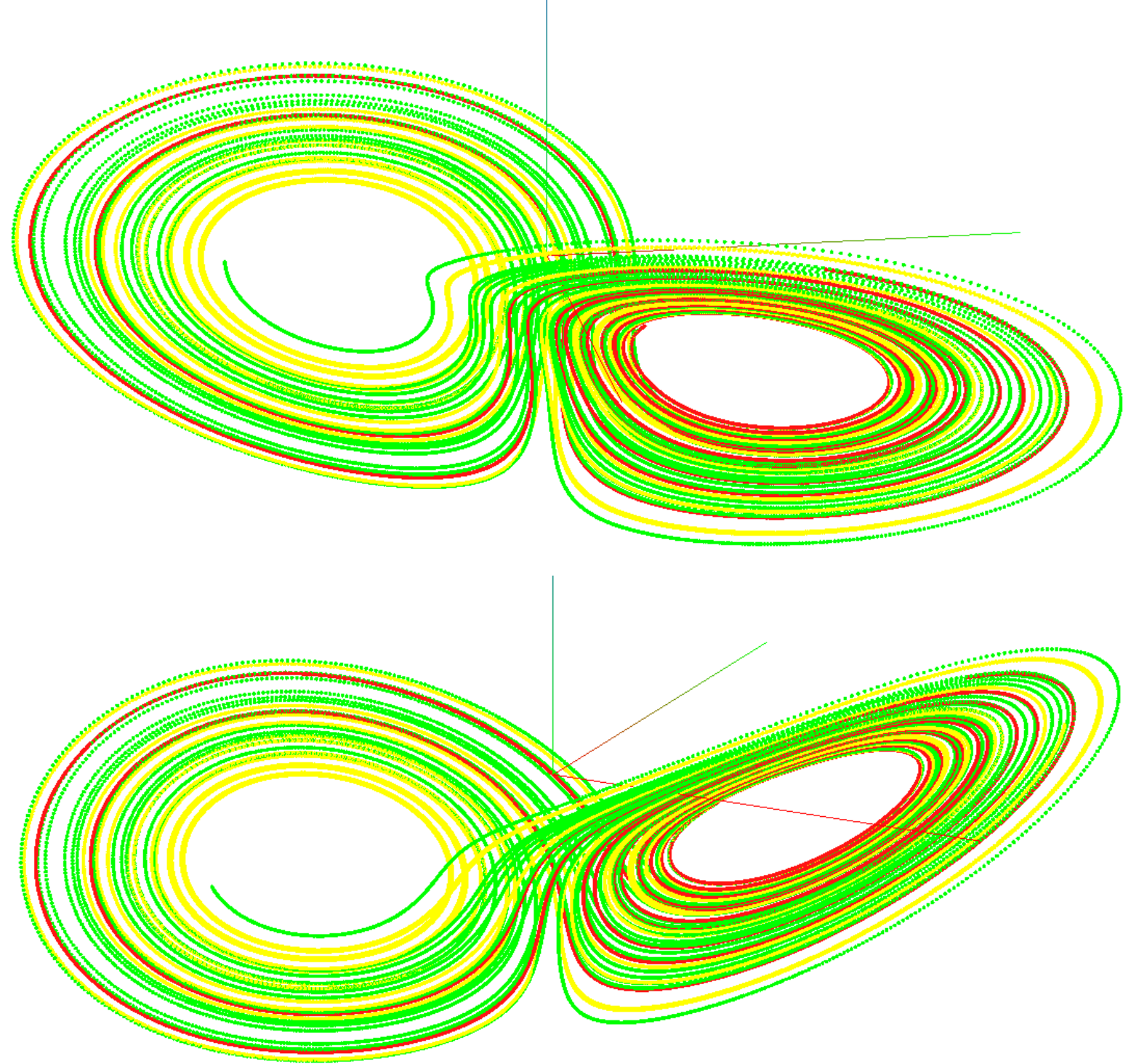

Рисунок 3 - Аттрактор Лоренца (первая, вторая, третья производные).

ISPC The Best of European Innovations, Gothenburg, Sweden 


\section{References:}

1. Gorodetskiy AS (2001) Minimal'nye attraktory i chastichno giperbolicheskie mnozhestva dinamicheskikh sistem. Diss. k. f.-m. n., MGU, 2001.

2. (2015) Galereya samykh strannykh attraktorov. LENTA.RU. Available: http://www.lenta.ru/photo/2013/03/28/visuali z ation/ (Accessed: 20.03.2015).

3. Nikul'chev EV (2007) Geometricheskiy metod rekonstruktsii sistem po eksperimental'nym dannym. Pis'ma v ZhTF. 2007. T. 33. Vyp. 6. pp. 83-89.

4. Nikul'chev EV (2010) Identifikatsiya dinamicheskikh sistem na osnove simmetriy rekonstruirovannykh attraktorov m. 2010. Available: http://nikulchev.ru/learnbook3.html (Accessed: 20.03.2015).

5. (2015) Attraktor Lorentsa. Available: http://rfwiki.org/\%D0\%90\%D1\%82\%D1\%82\% D1\%80\%D0\%B0\%D0\%BA\%D1\%82\%D0\%B E\%D1\%80 \%D0\%9B\%D0\%BE\%D1\%80\%D0
\%B5\%D0\%BD\%D1\%86\%D0\%B0 (Accessed: 20.03.2015).

6. Kuznetsov SP (2001) Sistema Lorentsa; Lektsiya 4. Dinamika sistemy Lorentsa. Dinamicheskiy khaos (kurs lektsiy). Moscow: Fizmatlit, 2001.

7. Saltzman B (1962) Finite amplitude free convection as an initial value problem. Journal of the atmospheric science, № 7, $1962-$ pp. 329-341.

8. Lorents E (1981) Determinirovannoe neperiodicheskoe dvizhenie. Strannye attraktory. - Moscow, 1981. — pp. 88-116.

9. (2015) Risuem prostye ob"ekty. Available: http://citforum.ru/programming/opengl/opengl 03.shtml (Accessed: 20.03.2015).

10. Shevtsov AN, Kaliyeva G (2014) SOME ASPECTS OF MODELING THE STRANGE ATTRACTORS OF LORENZ IN DELPHI. ISJ Theoretical \& Applied Science 10 (18): 6881. Soi: http://s-o-i.org/1.1/TAS*10(18)16 